\title{
Malária em Cruzeiro do Sul (Amazônia Ocidental brasileira): análise da série histórica de 1998 a 2008
}

\author{
Kleynianne Medeiros de Mendonça Costa, ${ }^{1,2}$ \\ Walquíria Aparecida Ferreira de Almeida, ${ }^{3}$ \\ Izanelda Batista Magalhães, ${ }^{4}$ Roberto Montoya, ${ }^{5}$ \\ Marco Sabóia Moura ${ }^{6}$ e Marcus Vinícius Guimarães de Lacerda ${ }^{1,6,7}$
}

Como citar Costa KMM, Almeida WAF, Magalhães IB, Montoya R, Moura MS, Lacerda MVG. Malária em Cruzeiro do Sul (Amazônia Ocidental brasileira): análise da série histórica de 1998 a 2008. Rev Panam Salud Publica. 2010;28(5):353-60.

RESUMO Objetivo. Descrever as características epidemiológicas da malária e seus principais determinantes no Município de Cruzeiro do Sul, no Estado do Acre, Brasil, entre 1998 e 2008.

Métodos. Este estudo descritivo, retrospectivo, utilizou dados secundários disponíveis nos sistemas de informação em malária desenvolvidos pelo Ministério da Saúde do Brasil (SISMAL/SIVEP-Malária). Os dados foram analisados no software TABLEAU ${ }^{\circledR}$. Dados geoespaciais foram obtidos para avaliar a distribuição dos casos de malária.

Resultados. A incidência parasitária anual (IPA) de 27 casos/1 000 habitantes em 1998 chegou a 571,5 casos/1 000 habitantes em 2006, quando a cidade registrou sua maior epidemia, subsequente ao estabelecimento de um programa estadual de incentivo à perfuração de tanques para piscicultura, em 2005. As localidades rurais apresentaram maior número de casos. Entretanto, as localidades periurbanas que possuíam tanques de piscicultura tiveram IPAs mais elevadas do que áreas sem tanques. Após a intensificação das ações do Programa Nacional de Controle da Malária, a IPA diminuiu para 152,9 casos/1 000 habitantes em 2008.

Conclusões. O incentivo a atividades econômicas em áreas periurbanas de transmissão instável de malária, típicas da América Latina, deve ser muito bem planejado. O controle da malária em Cruzeiro do Sul baseou-se em estratégias integradas implementadas simultaneamente pelos governos federal, estadual e municipal, como preconizado pelo Plano Nacional de Controle da Malária. É importante ressaltar a utilidade de um bom sistema de informação como o SIVEP-Malária para estimar a carga de doença e monitorar de forma eficiente o impacto das intervenções.

Palavras-chave Malária; estudos epidemiológicos; incidência; piscicultura; Brasil.

1 Universidade do Estado do Amazonas, Manaus, AM, Brasil. Correspondência: Marcus Vinícius Guimarães de Lacerda, marcuslacerda.br@gmail.com

2 Universidade Federal do Acre, Cruzeiro do Sul, AC, Brasil.

3 Ministério da Saúde, Brasília, DF, Brasil.

4 Secretaria de Estado de Saúde do Acre, Rio Branco, AC, Brasil.

5 Organização Pan-Americana da Saúde, Brasília, DF, Brasil.
Atualmente, a malária é a mais incidente doença parasitária do mundo. Estima-se que o número de casos novos

\footnotetext{
6 Fundação de Medicina Tropical do Amazonas, Manaus, AM, Brasil.

7 Centro Universitário Nilton Lins, Manaus, AM, Brasil.
}

a cada ano chegue a 250 milhões, com 880 mil mortes. Aproximadamente metade da população mundial está exposta à infecção, especialmente pessoas que vivem em países pobres (1).

Desde que o governo brasileiro se voltou para a organização dos planos de intervenção sanitária na Amazônia, essa 
área tem gerado grande preocupação social no Brasil (2). As condições socioeconômicas e o clima tropical úmido favorecem a proliferação de vetores de diversas doenças infecciosas na região, entre elas a malária (3). Dos casos notificados de malária no Brasil, 99,7\% ocorreram em estados que compõem a Amazônia Legal (Amazonas, Acre, Amapá, Rondônia, Roraima, Pará, Tocantins, Mato Grosso e Maranhão). Ao mesmo tempo, sabe-se que a malária não ocorre de forma homogênea, sendo influenciada por diferentes determinantes epidemiológicos, que variam fundamentalmente em função das formas de ocupação do solo e das modalidades de exploração econômica dos recursos naturais (4-6).

O Estado do Acre é o mais ocidental do Brasil. Possui 22 municípios dispostos ao longo dos rios Juruá e Acre-Purus (7). A partir do início da exploração da borracha, no final do século XIX, em especial nesse Estado, a incidência de malária teve significativo aumento, em função da intensa imigração de nordestinos sem exposição anterior a essa doença (4). A partir daí, elementos relacionados ao clima, à ocupação urbana não planejada e à intensa migração de pessoas de áreas rurais para áreas urbanas têm contribuído para o aumento do número de casos de malária na Amazônia Legal. Como reflexo dessa tendência, no ano de 2006, três municípios

FIGURA 1. Localização espacial de Cruzeiro do Sul, Acre, Brasil

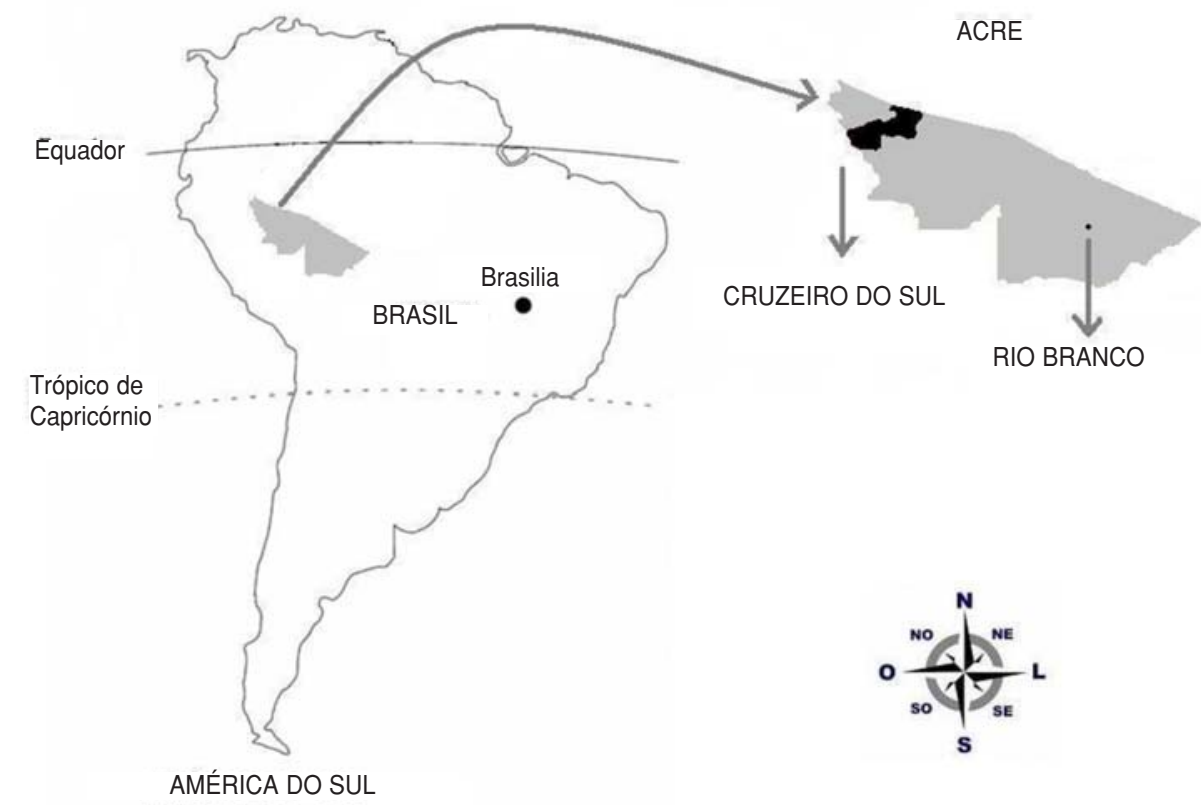

sozinhos contribuíram com 22,5\% dos casos de malária notificados no Brasil: Cruzeiro do Sul (oeste do Acre), Manaus (capital do Amazonas) e Porto Velho (capital de Rondônia) (8).

Em Cruzeiro do Sul, a incidência parasitária anual (IPA) em 2006 chegou a 571,5/1 000 habitantes, caracterizando essa área como hiperendêmica. As causas para tamanha endemicidade são mal compreendidas. Aventa-se a possibilidade de que, entre outros fatores, tanques destinados à piscicultura, construídos como resultado de um programa estadual de incentivo a essa atividade econômica, tenham-se comportado como criadouros permanentes de Anopheles darlingi, em especial na área periurbana.

O objetivo deste trabalho foi descrever as características epidemiológicas da malária no Município de Cruzeiro do Sul, no Estado do Acre, Brasil, bem como seus principais determinantes, entre 1998 e 2008, com base em informações secundárias extraídas de sistemas nacionais de informação sobre malária.

\section{MÉTODOS}

Trata-se de um estudo descritivo, de natureza retrospectiva, sobre a situação epidemiológica da malária no Município de Cruzeiro do Sul, no período de 1998 a 2008.
Cruzeiro do Sul está localizado na latitude $7^{\circ} 37^{\prime} 51^{\prime \prime} S$ e longitude $72^{\circ} 40^{\prime} 12^{\prime \prime} \mathrm{O}$, no Estado do Acre (9), às margens do Alto Rio Juruá (figura 1). Sua população em 2008 era estimada em 89095 habitantes (9). É o segundo maior município do Estado, depois da capital Rio Branco, com uma área de $7781,5 \mathrm{~km}^{2}$. Limita-se ao norte com o Estado do Amazonas, ao sul com o município acreano de Porto Valter, ao leste com o município acreano de Tarauacá e a Oeste com o Peru e os municípios acreanos de Mâncio Lima e Rodrigues Alves. Fica a cerca de 710 km de Rio Branco, por rodovia federal (BR-364). Apresenta clima quente e úmido, com chuvas frequentes e temperatura anual média de $26{ }^{\circ} \mathrm{C}$. Assim como os demais municípios da Amazônia Legal, apresenta apenas duas estações climáticas: a estação seca, que engloba principalmente os meses de maio a outubro, e a estação chuvosa, que ocorre nos demais meses do ano. $\mathrm{O}$ acesso terrestre, pela BR-364, à capital do estado, limita-se à estação seca. Nos outros meses, o fluxo de pessoas para o município acontece por via aérea ou fluvial, pelo Rio Juruá.

O extrativismo da borracha foi, até o início do século $\mathrm{XX}$, a atividade econômica de maior destaque desenvolvida em Cruzeiro do Sul. Atualmente, a farinha de mandioca é a base da economia. A municipalização das ações e serviços de saúde ainda não foi efetivada em Cruzeiro do Sul, ficando as ações de controle de endemias sob a responsabilidade da Secretaria de Estado de Saúde (SESACRE), vinculada ao Núcleo de Divisão de Endemias.

As variáveis analisadas neste estudo foram: número mensal de casos diagnosticados de malária por espécie de Plasmodium, IPA (calculada a partir do número de casos de malária por 1000 habitantes, utilizando dados do censo demográfico e estimativas do Instituto Brasileiro de Geografia e Estatística, IBGE), idade, sexo, gravidez, localidade provável de infecção, tipo de ambiente (rural ou urbano), número de óbitos por malária, distribuição espacial dos tanques de piscicultura na área urbana e índice pluviométrico mensal.

\section{Fonte de dados}

Os dados foram coletados a partir dos arquivos eletrônicos de notificação nacional de casos de malária da antiga Fundação Nacional de Saúde (FUNASA), do Sistema de Informação do Programa Na- 
cional de Controle da Malária (SISMAL), que funcionou de 1998 a 2002, e do Sistema de Informação de Vigilância Epidemiológica da Malária (SIVEP-Malária), da atual Secretaria de Vigilância em Saúde (SVS), em funcionamento desde 2003. Todos os dados apresentados foram coletados da base de informações atualizada até março de 2009.

O SIVEP-Malária é uma versão mais atualizada do antigo SISMAL, dispondo de mais variáveis e podendo ser consultado online, com acesso por senha, por profissionais que trabalham no controle da doença. $\mathrm{O}$ banco de dados utilizado para análise durante o período foi obtido junto à SESACRE e ao Ministério da Saúde, após assinatura de documento que garantiu a confidencialidade da informação por parte dos pesquisadores.

Para as localidades urbanas cadastradas previamente no SIVEP-Malária, realizou-se a tomada de coordenadas geográficas por georreferenciamento (com o uso de aparelho de Global Positioning System, GPS) em ponto central da localidade (igrejas ou associações de bairro), bem como nas margens de açudes ou escavações com coleção hídrica, destinados à piscicultura nessas mesmas localidades urbanas em 2006.

O número de óbitos por malária foi obtido do Sistema de Informação de Mortalidade (SIM), do Banco de Dados do Sistema Único de Saúde (DATASUS) (disponível até 2006) (10). O índice pluviométrico (em mm de chuva) mensal de Cruzeiro do Sul foi obtido durante todos os anos do estudo do Instituto Nacional de Meteorologia (INMET), na Estação Meteorológica do $61^{\circ}$ Batalhão de Infantaria e Selva.

Todos os dados foram analisados no programa TABLEAU ${ }^{\circledR}$ versão 3.1. As curvas de tendência foram calculadas por meio do coeficiente de correlação de Pearson, com nível de significância para $P<0,05$. O projeto foi aprovado pelo Comitê de Ética em Pesquisa da Fundação de Medicina Tropical do Amazonas (processo 0655/2008-FMT-AM), com isenção do termo de consentimento livre e esclarecido (CAAE $n^{\circ}$ 0006.0.114.114-08).

\section{RESULTADOS}

No período de 1998 a 2008, foram notificados em Cruzeiro do Sul 157647 casos de malária, com instalação de uma epidemia no início do ano de 2004 (tabela 1). Ao longo da série histórica, notase um discreto aumento na proporção de mulheres acometidas, um pequeno número de casos de infecções mistas detectadas à microscopia ótica e tendência de decréscimo de infecções por Plasmodium falciparum, principalmente a partir de 2007. Nota-se também que cerca da metade dos casos de malária $(45,2 \%)$ foi notificada entre escolares e pré-escolares (crianças com menos de 14 anos de idade). Do total dos exames positivos confirmados em mulheres entre 2003 e 2008 (informação disponível apenas no SIVEP-Malária), 2,6\% estavam relacionados a pacientes grávidas. Desses casos, $32,1 \%$ foram causados por $P$. falciparum (dados não apresentados).
A figura 2 ilustra mais claramente o aumento do número de casos em 2004 e do número total de casos de malária por $P$. falciparum e Plasmodium vivax (P. vivax), sempre predominando esse último. Além disso, é possível identificar que, a partir de 2004, Cruzeiro do Sul passou a ser responsável por mais de $50,0 \%$ dos casos de malária registrados no Estado do Acre.

A figura 3 mostra a tendência de aumento da infecção por $P$. vivax e de diminuição da infecção por $P$. falciparum, porém sem significância estatística. Os períodos de maior transmissão da doença em cada ano foram heterogêneos nos anos estudados, não caracterizando, portanto, sazonalidade característica (dados não apresentados). Também não se verificou associação da incidência de malária com os índices pluviométricos (dados não apresentados).

Levando-se em consideração o local provável de infecção, os municípios que mais contribuíram para o registro de casos em Cruzeiro do Sul foram: Guajará (Estado do Amazonas), com 5,4\%; Rodrigues Alves (Estado do Acre), com 4,9\%; Mâncio Lima (Acre), com 2,1\%; e Tarauacá (Acre), com 2,0\%. Durante o período estudado, não houve mudança na proporção de casos de malária diagnosticados em outros municípios.

Foram mapeados 179 açudes ou escavações com coleção de água, todos na zona periurbana de Cruzeiro do Sul. A figura 4 mostra um mapa hidrográfico da área urbana da cidade e suas redondezas. Na figura 4A observa-se o mapa de 2004,

TABELA 1. Série histórica dos casos de malária diagnosticados no Município de Cruzeiro do Sul (AC), Brasil, 1998 a 2008

\begin{tabular}{|c|c|c|c|c|c|c|c|c|c|c|c|c|c|c|c|}
\hline \multirow[b]{3}{*}{ Ano } & \multirow[b]{3}{*}{ População } & \multirow[b]{3}{*}{ Positivos } & \multirow{3}{*}{$\begin{array}{c}\text { Mulheres } \\
(\%)\end{array}$} & \multirow{3}{*}{$\begin{array}{c}\text { Homens } \\
(\%)\end{array}$} & \multirow{2}{*}{\multicolumn{2}{|c|}{ Plasmodium }} & \multirow[b]{3}{*}{$P f / P v^{a}$} & \multirow[b]{3}{*}{$\mathrm{IPA}^{\mathrm{b}}$} & \multirow[b]{3}{*}{$\mathrm{IFA}^{\mathrm{C}}$} & \multicolumn{4}{|c|}{ Idade (anos) } & \multirow{3}{*}{$\begin{array}{c}\text { Urbano } \\
(\%)\end{array}$} & \multirow{3}{*}{$\begin{array}{l}\text { No. de } \\
\text { óbitos }\end{array}$} \\
\hline & & & & & & & & & & \multirow{2}{*}{$\begin{array}{l}<1 \\
(\%)\end{array}$} & \multirow{2}{*}{$\begin{array}{c}1 \text { a } 4 \\
(\%)\end{array}$} & \multirow{2}{*}{$\begin{array}{c}5 \text { a } 14 \\
(\%)\end{array}$} & \multirow{2}{*}{$\begin{array}{l}>14 \\
(\%)\end{array}$} & & \\
\hline & & & & & falciparum & vivax & & & & & & & & & \\
\hline 1998 & 60817 & 1645 & $\mathrm{ND}^{\mathrm{d}}$ & $N^{d}$ & 489 & 1152 & 4 & 27,0 & 29,7 & $N D^{d}$ & $N D^{d}$ & $N D^{d}$ & $N^{d}$ & $\mathrm{ND}^{\mathrm{d}}$ & 4 \\
\hline 1999 & 62691 & 6195 & 40,0 & 59,9 & 1863 & 4293 & 39 & 98,8 & 30,1 & 2,7 & 15,1 & 25,9 & 56,3 & $N D^{d}$ & 5 \\
\hline 2000 & 67441 & 5428 & 35,7 & 64,3 & 1418 & 3991 & 18 & 80,5 & 26,1 & 1,7 & 20,1 & 27,9 & 50,2 & $N D^{d}$ & 6 \\
\hline 2001 & 69772 & 2070 & 33,7 & 66,3 & 424 & 1638 & 8 & 29,7 & 20,5 & 1,1 & 21,0 & 29,2 & 48,7 & $N D^{d}$ & 0 \\
\hline 2002 & 71571 & 1996 & 33,6 & 66,4 & 485 & 1508 & 3 & 27,9 & 24,3 & 0,2 & 20,1 & 30,8 & 48,9 & $N D^{d}$ & 4 \\
\hline 2003 & 78344 & 3774 & 43,1 & 56,9 & 897 & 2874 & 3 & 48,2 & 23,8 & 2,5 & 10,8 & 28,6 & 58,0 & 26,4 & 3 \\
\hline 2004 & 80006 & 16254 & 44,3 & 55,7 & 3394 & 12852 & 8 & 203,2 & 20,9 & 2,7 & 9,9 & 27,9 & 59,4 & 30,5 & 3 \\
\hline 2005 & 81180 & 30715 & 44,7 & 55,3 & 7443 & 23244 & 28 & 378,4 & 24,2 & 2,5 & 9,8 & 28,6 & 59,1 & 27,3 & 6 \\
\hline 2006 & 83345 & 47628 & 44,3 & 55,7 & 15714 & 31789 & 125 & 571,5 & 33,0 & 3,1 & 9,9 & 30,0 & 56,9 & 23,3 & 3 \\
\hline 2007 & 78694 & 28323 & 44,8 & 55,2 & 5279 & 23018 & 26 & 359,9 & 18,6 & 3,1 & 10,3 & 30,3 & 56,3 & 19,8 & 0 \\
\hline 2008 & 89095 & 13619 & 44,4 & 55,6 & 2522 & 12791 & 90 & 152,9 & 16,4 & 2,3 & 14,3 & 29,8 & 53,4 & 16,5 & 0 \\
\hline Total & $N A^{d}$ & 157647 & 40,9 & 59,1 & 39928 & 119150 & 352 & 179,8 & 24,3 & 2,2 & 14,1 & 28,9 & 54,7 & 24,0 & 34 \\
\hline
\end{tabular}

\footnotetext{
a Infecções mistas ( $P$. falciparum + $P$. vivax).

b IPA = incidência parasitária anual.

c IFA = Incidência de $P$. falciparum.

${ }^{d} \mathrm{NA}$ = não se aplica; ND = não-disponível
} 
FIGURA 2. Casos de malária por ano e espécie parasitária no Município de Cruzeiro do Sul e percentual de casos em relação ao Estado do Acre, Brasil, 1998 a 2008

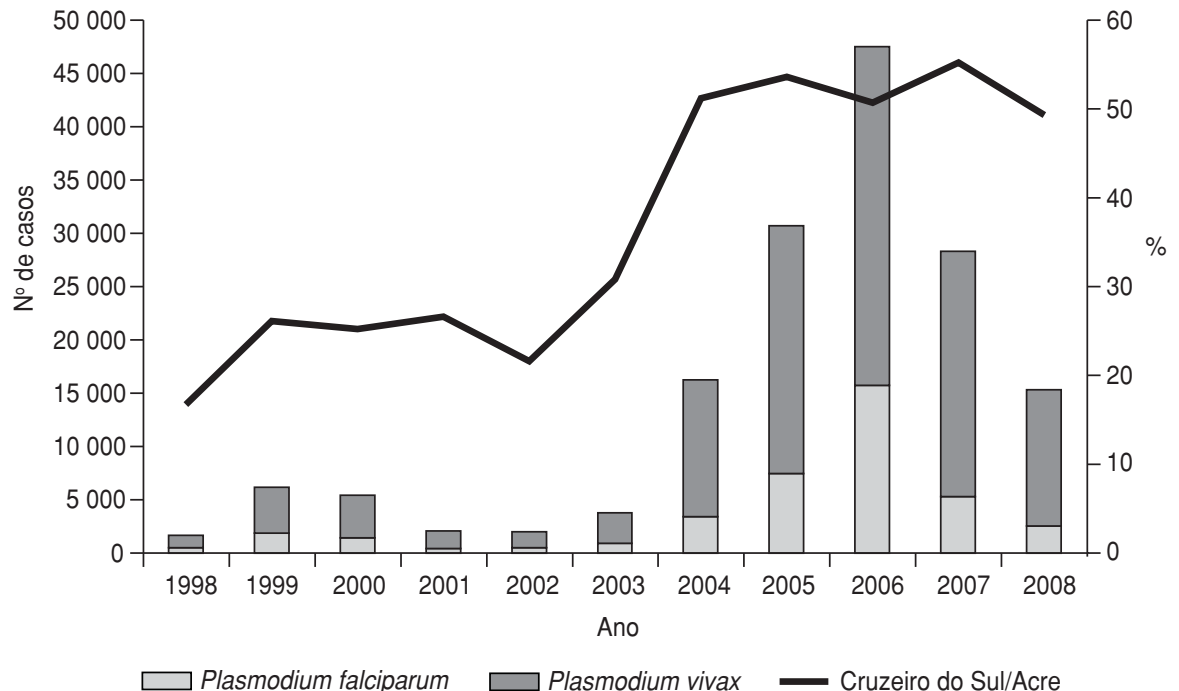

FIGURA 3. Percentual de casos de malária por ano e espécie parasitária no Município de Cruzeiro do Sul e curvas de tendência, Estado do Acre, Brasil, 1998 a 2008

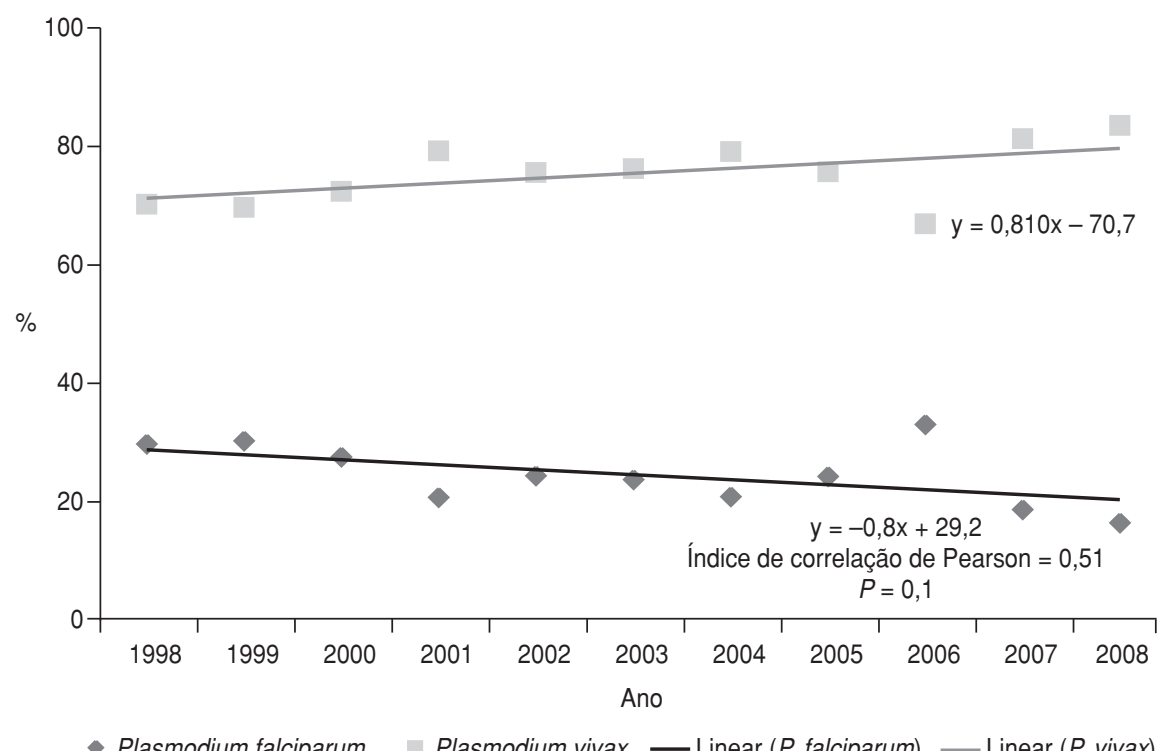

momento imediatamente anterior ao incentivo governamental à escavação de tanques para piscicultura no município, com a IPA de cada localidade. A figura 4B mostra um mapa de 2006, quando os 179 açudes já estavam escavados. As áreas com IPA elevada tenderam a concentrar-se perto de açudes ou em áreas próximas a rios e igarapés.

\section{DISCUSSÃO}

A análise crítica de dados secundários, muitas vezes colhidos e não analisados apropriadamente, pode servir como base butilizadas. O preenchimento do SIVEPMalária é obrigatório para todos os casos suspeitos de malária. No Brasil, o tratamento só é administrado gratuitamente em caso de confirmação diagnóstica, e a venda de antimaláricos em farmácias não é permitida.

$\mathrm{Na}$ Amazônia Brasileira, os cenários de transmissão da malária são diversos, porque a doença possui característica "glolocal" (termo sociológico utilizado para se referir a problemas globais que apresentam características locais). Os determinantes da malária estão relacionados aos três componentes da doença: a população suscetível, a presença do agente etiológico e a presença do vetor. Assim, a análise da situação epidemiológica de uma região não permite conclusões para as demais, na mesma Amazônia Legal, mas pode ajudar a compreender de que maneira inúmeros fatores, inclusive certas atividades econômicas, interferem no perfil epidemiológico da malária nos distintos estados da federação, com culturas distintas.

São múltiplos os possíveis fatores que contribuíram para que a IPA em Cruzeiro do Sul passasse de 27,0/1 000 habitantes em 1998 para 571,5/1 000 habitantes em 2006. Na realidade, apesar de a grande epidemia de malária em Cruzeiro do Sul ter-se iniciado em 2004, as IPAs dos anos 1999 e 2000 (98,8 e 80,5/ 1000 habitantes, respectivamente) já permitiam caracterizar a Cidade como local de alto risco para malária.

No Acre, em 2001, houve uma queda de $67,2 \%$ na incidência geral de malária e de $76,0 \%$ na incidência de $P$. falciparum em comparação a 1999. Isso aconteceu mesmo sendo o Acre o único estado da Amazônia Legal a centralizar suas ações em nível estadual, diferentemente dos outros estados, que adotavam a municipalização (12) — o que, em tese, tenderia a melhorar o controle da doença. Entretanto, a redução temporária da IPA observada nos anos de 2001 a 2003 no Acre foi apenas um reflexo do Plano de Intensificação das Ações de Controle da Malária (PIACM), iniciado em 2000 em toda a Amazônia Legal (exceto no Amapá, onde se iniciou em 2001), cujas ações não demonstraram efetividade sustentável em nenhum dos estados malarígenos após o ano de 2003.

Pelo aumento do número de mulheres acometidas ao longo do período de estudo, bem como pelo grande número de 
FIGURA 4. Mapa hidrográfico de Cruzeiro do Sul e redondezas, Estado do Acre, Brasil

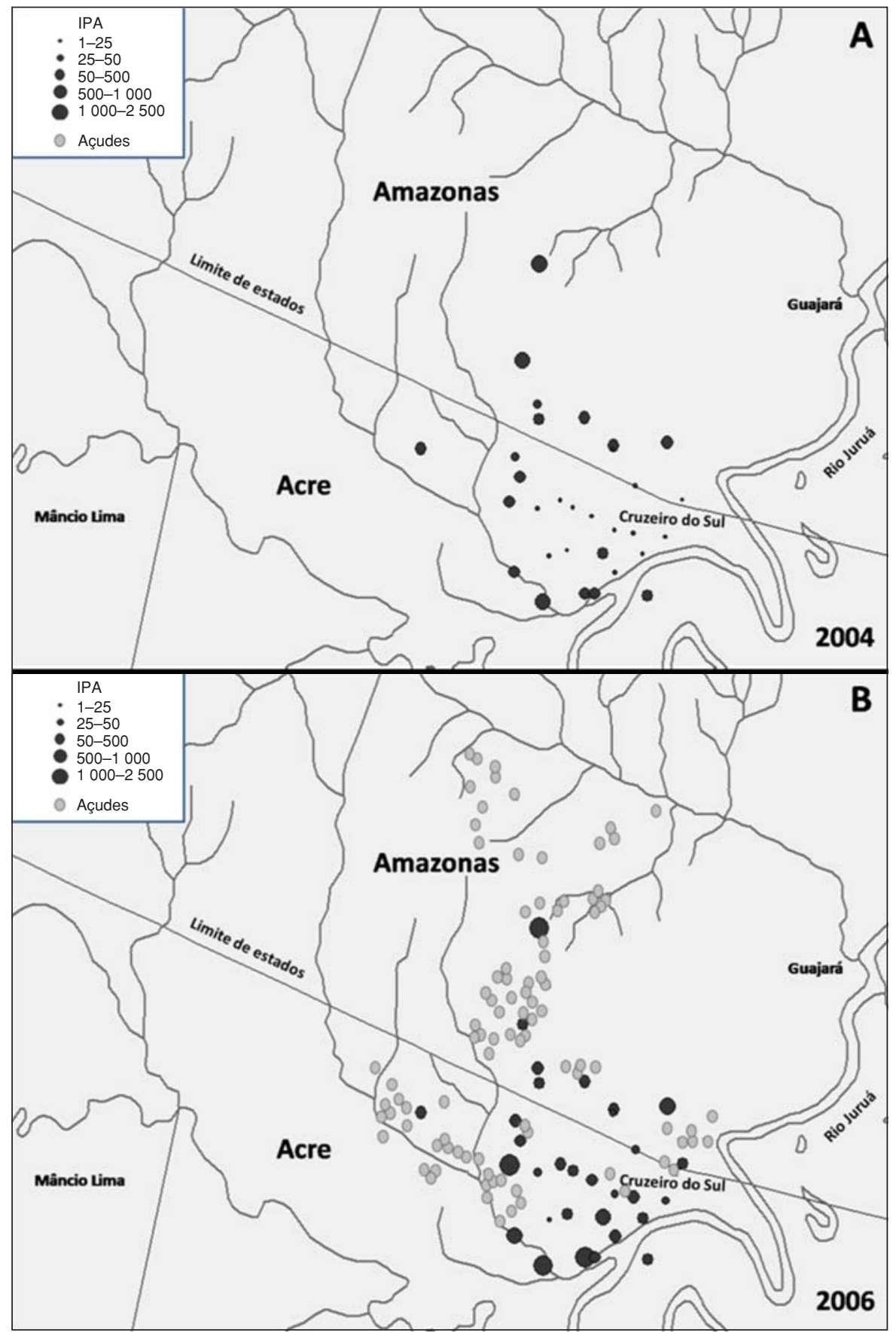

A) Incidência parasitária anual (pontos pretos) nas 32 localidades urbanas (cadastradas pelo SIVEP-Malária) em 2004, antes da escavação dos tanques de piscicultura. B) Incidência parasitária anual (pontos pretos) nas mesmas localidades da área urbana em 2006, com a geo-espacialização dos tanques de piscicultura, cuja escavação se iniciou em 2005 (pontos cinza).

crianças abaixo de 14 anos, é possível especular que a transmissão no Município esteja ocorrendo nas imediações do domicílio. É importante considerar que a média de infecção por $P$. falciparum em mulheres gestantes foi superior à média geral de infecção por $P$. falciparum $(32,1 \%$ vs. 22,6\%) no período de 2003 a 2008. Esse achado é compatível com a obser- vação de que gestantes são mais acometidas por essa espécie, em comparação com mulheres não gestantes, segundo estudo realizado no Município de Coari (Amazonas) (13).

Outros fatores determinantes da alta incidência de malária em Cruzeiro do Sul são: proximidade das comunidades à floresta, trânsito contínuo de pessoas in- fectadas vindas de outros municípios do Acre e do Amazonas, considerados malarígenos, e dificuldades operacionais do sistema de vigilância local (2). Não menos importantes são as condições trabalhistas sob as quais são contratados os responsáveis pelo controle da malária e outras doenças de transmissão vetorial, em regimes temporários não superiores a 1 ano, sem a perspectiva de um plano de cargos, carreiras e salários (14).

De forma peculiar, Cruzeiro do Sul, no período de julho a outubro, quando a estrada que liga esse município à capital Rio Branco (BR-364) encontra-se trafegável, passa a receber um elevado número de pessoas provenientes principalmente da capital e também de Rondônia, estado que concentra em sua capital, Porto Velho, altas incidências da doença, com semelhantes características de malária urbana e periurbana (15). Além disso, a festa religiosa da padroeira da cidade, que acontece em agosto, resulta no aumento do fluxo de pessoas da área rural para a área urbana. Esses fatores podem interferir no aumento da incidência de malária não só em Cruzeiro do Sul, mas também em municípios e/ou estados circunvizinhos, que em muito refletem a situação epidemiológica de Cruzeiro do Sul, pelo elevado trânsito diário de pessoas entre esses municípios, em especial Guajará, Mâncio Lima e Rodrigues Alves.

O aumento da malária em Cruzeiro do Sul entre 2004 e 2006 aconteceu mais no ambiente rural, mas também houve expressivo crescimento na área considerada urbana. Apesar de a malária ter classicamente maior impacto sobre as populações rurais (16-18), observa-se, na atualidade, com o intenso êxodo rural de tendência mundial, uma dinâmica de transmissão mais periurbana, em ambiente com boa receptividade, fundamentalmente pela ocupação desordenada, em áreas próximas à floresta tropical, com condições sanitárias e de instrução precárias (6). Situação semelhante pode ser vista na capital do Amazonas, Manaus (19).

O maior aumento proporcional de casos de malária em área rural pode se justificar pelo diagnóstico mais tardio e consequente perpetuação do ciclo de transmissão da doença. Na área urbana, o aumento da malária pode se dar pela intensa migração de pessoas que habitam em áreas rurais, mas também pelo incentivo a atividades econômicas de 
alto impacto ambiental, tais como o desmatamento para o estabelecimento de pastagens e a escavação de açudes para piscicultura, atividade que foi subsidiada pelo Governo do Estado do Acre em 2005.

Em 2004, existiam poucos tanques para piscicultura na área urbana de Cruzeiro do Sul. A partir de 2005, novos tanques foram escavados, coincidindo, em 2006, com as áreas de maior incidência de malária na área periurbana. Essas coleções de água parada se transformam em criadouros permanentes de anofelinos quando não existe a preocupação com a limpeza de suas margens (20). Com a escassez de recursos financeiros permanentes para sustentar a atividade de piscicultura, muitos tanques foram abandonados. A área de influência do tanque, entretanto, pode ser mais abrangente do que aquela que se observa na figura $4 \mathrm{~B}$, tendo em vista que a divisão das localidades registradas no SIVEP-Malária é heterogênea, e o georreferenciamento aconteceu em áreas centrais de maior aglomeração populacional. Um estudo realizado em Manaus, com espacialização dos tanques de piscicultura e dos casos de malária, individualmente, permitiu observar uma situação semelhante à encontrada em nosso estudo. Isso sugere que tais coleções de água, sem correnteza, são potenciais criadouros de anofelinos (21), o que poderia inclusive explicar a ausência de sazonalidade da doença em Cruzeiro do Sul. O estímulo a atividades econômicas como a piscicultura é recente na Amazônia brasileira e acontece sem o devido planejamento. Portanto, é possível que em muitas outras localidades da Amazônia essa nova atividade econômica esteja contribuindo para a perpetuação e o incremento dos indicadores da endemia malárica, em especial na área periurbana.

A análise da participação de Cruzeiro do Sul no percentual global de casos do estado aponta-o como principal notificante de casos de malária, representando mais de 50,0\% das notificações durante um período de 4 anos consecutivos, o que motivou o Governo do Estado do Acre, em parceria com a Prefeitura de Cruzeiro do Sul e o Ministério da Saúde, a partir de 2005, a elaborar um plano de intensificação das ações de controle à doença.

As principais estratégias de intensificação das ações de controle foram: 1) implantação de atividades pedagógicas que permitiram a mudança de compor- tamento e reforçaram a participação comunitária na adesão ao tratamento e na análise dos fatores de risco das populações afetadas $(22,23) ; 2)$ contratação e qualificação dos profissionais envolvidos no controle (treinamento de microscopistas, agentes comunitários de saúde, médicos, enfermeiros e demais profissionais); 3) compra de novos microscópios; 4) compra de testes rápidos para diagnóstico, exclusivamente para áreas remotas, de difícil acesso $\left(\right.$ Optimal ${ }^{\circledR}$, DiaMed, EUA); 5) compra de carros para deslocamento de equipes; 6) sistematização das buscas ativas para detecção de portadores assintomáticos de plasmódio; 7) construção e reforma de novos postos de diagnóstico, com aumento de 18 postos em 2003 para 29 postos em 2006, com três microscopistas nos postos de maior demanda; 8) planejamento estratégico de borrifações intradomiciliares e controle ambiental de criadouros; 9) introdução, em 2006, da combinação artesunato/mefloquina (Farmanguinhos ${ }^{\circledR}$, Fiocruz, Rio de Janeiro, RJ) como primeira opção de tratamento para malária por $P$. falciparum, que chegou a ser responsável por $36,0 \%$ dos casos nesse mesmo ano; 10) distribuição de mosquiteiros impregnados de longa duração, a partir de dezembro de 2007, em algumas comunidades do município.

Toda essa estratégia integrada de controle resultou na diminuição da IPA de 571,5 casos/1000 habitantes em 2006 para 152,9 casos/1000 habitantes em 2008. A melhoria da rede de diagnóstico e tratamento foi, de fato, uma ferramenta importante para a redução da morbimortalidade por malária $(24,25)$. Deve-se ressaltar ainda que, mesmo com o aumento explosivo do número de casos e com o aumento do percentual de $P$. falciparum, a letalidade total nunca foi superior a $0,02 \%$, o que vem ao encontro da redução da mortalidade por malária em todo o país nas últimas décadas. Sob certo aspecto, isso reflete a relativa eficácia de algumas ações de controle (26). No período estudado, de 11 anos estudados, houve uma tendência de diminuição da infecção por $P$. falciparum, ainda que não significativa, provavelmente em função da maior queda apenas nos últimos 2 anos, depois da introdução de artesunato/mefloquina, ainda que o impacto isolado dessa medida não possa ser avaliado com base nos dados apresentados.

Vale notar que, em 2006, os dois municípios que registraram maior incidên- cia de malária no Brasil se localizavam justamente na fronteira de Cruzeiro do Sul, Rodrigues Alves e Mâncio Lima, com 1619,1 e 1216,9 casos/1 000 habitantes, respectivamente. Isso revela que a dinâmica de transmissão da malária em Cruzeiro do Sul não é um fenômeno isolado e pontual, mas dependente de condições macrorregionais, motivo pelo qual as estratégias de controle devem ser adotadas sempre de maneira regionalizada, independentemente das adversidades políticas e das fronteiras geográficas.

As atividades econômicas na Amazônia estão diretamente ligadas aos cenários de transmissão da malária desde o século XIX, em que a exploração da borracha, mais marcante no Acre, proporcionou a perpetuação da malária como uma endemia regional. Desde a Campanha de Erradicação da Malária, iniciada em 1965, e baseada no uso do dicloro-difenil-tricloroetano (DDT), os desafios para o controle dessa doença nunca foram menores (27). Novas atividades econômicas ou de ocupação da terra devem ser planejadas conjuntamente com os órgãos responsáveis pelo controle de endemias, diálogo nem sempre fácil, pelo hábito do trabalho não interdisciplinar (28).

É preciso destacar a importância do bom sistema de informação sobre malária no Brasil, fruto do esforço do Ministério da Saúde, que aperfeiçoou o antigo SISMAL, proporcionando, com o SIVEPMalária, uma ferramenta eficaz para o planejamento das ações de controle da doença, mas que pode ter uma utilidade ainda maior se os municípios e estados sistematizarem a análise das suas informações. A disponibilidade online de dados e relatórios permite maior acesso à informação, e a possibilidade de análise estatística do banco de dados com softwares específicos compatíveis são elos importantes na parceria entre o serviço de saúde e a universidade. Tal sistema permite inclusive a detecção precoce de epidemias, o que pode ter valor inestimável na sua prevenção (29).

O presente estudo tem limitações, destacando-se entre elas: o uso de dados secundários, sujeitos à sub-notificação; a impossibilidade de aferir adequadamente a contribuição de cada medida de controle adotada em Cruzeiro do Sul, em função de não ter havido um desenho experimental apropriado para a avaliação de impacto; a natureza ecológica da ava- 
liação; a falta de evidências para embasar a hipótese dos tanques de piscicultura como provável fator determinante da perpetuação da doença no município. Sobre esse último aspecto, outros estudos controlados devem avaliar o real impacto dos tanques na transmissão da malária, incluindo a vigilância vetorial e o georreferenciamento de casos individuais autóctones da doença, com análise espacial mais robusta.

Em conclusão, o artigo descreveu as principais características epidemiológicas da malária no Município de Cruzeiro do Sul, no Estado do Acre, entre 1998 e
2008, período marcado por uma grande epidemia de malária da região. É nossa hipótese que os principais determinantes da doença nessa região estiveram relacionados aos processos migratórios, à falta de articulação entre os vários níveis de governo e à construção de tanques para piscicultura como parte de um projeto de incentivo econômico com planejamento falho. As diversas medidas tomadas, entretanto, em resposta ao aumento expressivo do número de casos, mostraram que o controle da doença é factível na Amazônia Brasileira, apenas com base na intensificação do controle, em que pesem o diagnóstico e o tratamento precoces e o estabelecimento de parcerias entre os diferentes níveis de governo. O impacto do uso de testes rápidos, combinação de antimaláricos com derivados de artemisininas no tratamento de malária falciparum e mosquiteiros impregnados não pode ser negligenciado, apesar da necessidade de mais estudos de impacto na América Latina. Os dados do sistema de informação SIVEP-Malária devem ser analisados de forma mais direcionada e crítica, permitindo assim o reconhecimento precoce de epidemias iminentes.

\section{REFERÊNCIAS}

1. World Health Organization. World Malaria Report 2009. Disponível em: http:/ / whqlibdoc. who.int/publications/2009/9789241563901_ eng.pdf. Acessado 18 de abril de 2009.

2. Confalonieri UEC. Saúde na Amazônia: um modelo conceitual para a análise de paisagens e doenças. Estud Av. 2005;53(19):221-36.

3. Tauil PL. The status of infectious disease in the Amazon region. Emerg Infect Dis. 2009; 15(4):625.

4. Cesário M, Cesário RR. Malária, Amazônia e desenvolvimento. Sci American Brasil. 2006; 54:54-5.

5. Barata RB. Malária no Brasil: panorama epidemiológico na última década. Cad Saude Publica. 1995;11(1):128-36.

6. Marques AC. Migration and the dissemination of malaria in Brazil. Mem Inst Oswaldo Cruz. 1996;81(suppl 2):17-30.

7. Governo do Estado do Acre, Secretaria de Estado de Ciência, Tecnologia e Meio Ambiente. Zoneamento ecológico-econômico: recursos naturais e meio ambiente. Rio Branco: SECTMA; 2000.

8. Brasil, Ministério da Saúde. Situação epidemiológica da malária no Brasil 2006. Disponível em: http://bvsms.saude.gov.br/bvs/ publicacoes/folder_malaria_2006_web.pdf. Acessado em 18 de fevereiro de 2008.

9. Instituto Brasileiro de Geografia e Estatística. Censo Demográfico 2000. Disponível em: http://www.ibge.gov.br/home/estatistica/ populacao/censo2000/default.shtm. Acessado em 29 de outubro de 2006.

10. Brasil, Ministério da Saúde. DATASUS 2009. Disponível em: http://www2.datasus.gov. $\mathrm{br} / \mathrm{DATASUS} /$ index.php?area=02. Acessado em 30 de abril de 2009 .

11. Barata Barradas RC. O desafio das doenças emergentes e a revalorização da epidemiologia descritiva. Rev Saude Publica. 1997;31(5): 531-7.
12. Tauil PL. Avaliação de uma nova estratégia de controle da malária na Amazônia Brasileira [tese]. Brasília: Universidade de Brasília; 2002.

13. Martínez-Espinosa FE, Daniel-Ribeiro CT, Alecrim WD. Malaria during pregnancy in a reference centre from the Brazilian Amazon: unexpected increase in the frequency of Plasmodium falciparum infections. Mem Inst Oswaldo Cruz. 2004;99(1):19-21.

14. Tauil PL. Perspectivas de controle de doenças transmitidas por vetores no Brasil. Rev Soc Bras Med Trop. 2006;39(3):275-7.

15. Gil LH, Tada MS, Katsuragawa TH, Ribolla PE, da Silva LH. Urban and suburban malaria in Rondonia (Brazilian Western Amazon) II. Perennial transmissions with high anopheline densities are associated with human environmental changes. Mem Inst Oswaldo Cruz. 2007;102(3):271-6.

16. Suaréz-Mutis MC, Coura JR. Mudanças no padrão epidemiológico da malária em área rural do médio Rio Negro, Amazônia Brasileira, análise retrospectiva. Cad Saude Publica. 2007;23(4):795-804.

17. Varga ID. Fronteiras da urbanidade sanitária: sobre o controle da malária. Saúde Soc. 2007; 16(1):28-44.

18. Silva-Nunes $M$, Malafronte RS, Luz BA, Souza EA, Martins LC, Rodrigues SG, et al. The Acre Project: the epidemiology of malaria and arthropod-borne virus infections in a rural Amazonian population. Cad Saude Publica. 2006;22(6):1325-34.

19. Gonçalves MJF, Alecrim WD. Non-planned urbanization as a contributing factor for malaria incidence in Manaus-Amazonas, Brazil. Rev Salud Publica. 2004;6(2):156-66.

20. Ferrete JA, Lemos JC, Lima SC. Lagos artificiais e os fatores condicionantes e determinantes no processo saúde-doença. Caminhos Geogr. 2004;5(13):187-200.
21. Terrazas WCM. Desenvolvimento de SIG para análise epidemiológica da distribuição espacial da malária no Município de Manausum enfoque em nível local [dissertação]. Rio de Janeiro: Escola Nacional de Saúde Pública da Fiocruz; 2005.

22. Rocha MNA, Ferreira EAP, Souza JM. Aspectos históricos da malária. Rev Para Med. 2006; 20(3):81-2.

23. Rocha MNA, Pereira EAP, Souza JM. Uma proposta de prevenção e controle da malária em pequenas comunidades. Rev Para Med. 2005;19(4):47-51.

24. Tauil PL. Controle de doenças transmitidas por vetores no sistema único de saúde. Inf Epidemiol SUS. 2002;11(2):59-60.

25. Cordeiro CES, Filomeno CRM, Costa CMA, Couto AARD. Perfil epidemiológico da malária no Estado do Pará em 1999 com base em uma série histórica de dez anos (19891999). Inf Epidemiol SUS. 2002;11(2):69-77.

26. Penna G, Pinto LF, Soranz D, Glatt R. High incidence of diseases endemic to the Amazon region of Brazil, 2001-2006. Emerg Infect Dis. 2009;15(4):626-32.

27. Loiola CC, Silva CJ, Tauil PL. Controle da malária no Brasil: 1965 a 2001. Rev Panam Salud Publica. 2002;11(4):235-44.

28. Albuquerque BC. Reorganization of the Brazilian Amazon region and malaria control. Mem Inst Oswaldo Cruz. 1992;87 Suppl 3:341.

29. Braz RM, Andreozzi VL, Kale PL. Detecção precoce de epidemias de malária no Brasil: uma proposta de automação. Epidemiol Serv Saúde. 2006;15(2):21-33.

Manuscrito recebido em 7 de setembro de 2009. Aceito em versão revisada em 29 de junho de 2010. 
ABSTRACT Objective. To describe the epidemiological characteristics of malaria and its main determinants in the municipality of Cruzeiro do Sul, State of Acre, Brazil, between 1998 and 2008.

Malaria in Cruzeiro do Sul

Methods. This descriptive, retrospective study was carried out with secondary data (Western Brazilian Amazon): analysis of the historical series from 1998 to 2008 available from the malaria information systems developed by the Brazilian Ministry of Health (SISMAL/SIVEP-Malária). The data were analyzed using the TABLEAU ${ }^{\circledR}$ software. Geospatial data were obtained to assess the distribution of malaria cases. Results. The annual parasite incidence (API) of 27 cases/1 000 population in 1998 reached 571.5 cases $/ 1000$ population in 2006, the year in which the city recorded its largest epidemics, following the establishment of a state program that encouraged the digging of tanks for fish farming in 2005. Rural sites had the highest number of cases. However, peri-urban locations with fish tanks had higher APIs than peri-urban areas without tanks. Following the strengthening of control actions by the National Malaria Control Program, the API in Cruzeiro do Sul dropped to 152.9 cases / 1000 population in 2008.

Conclusions. The type of economic activity fostered in peri-urban areas characterized by unstable malaria transmission, which are typical of Latin America, must be very well planned. Malaria control in Cruzeiro do Sul relied on integrated strategies implemented simultaneously by federal, state, and city governments, as recommended by the National Malaria Control Program. It is important to underscore the usefulness of a reliable information system such as SIVEP-Malária to estimate the burden of disease and efficiently monitor the impact of interventions.

Key words Malaria; epidemiologic studies; incidence; control; fish culture; Brazil. 


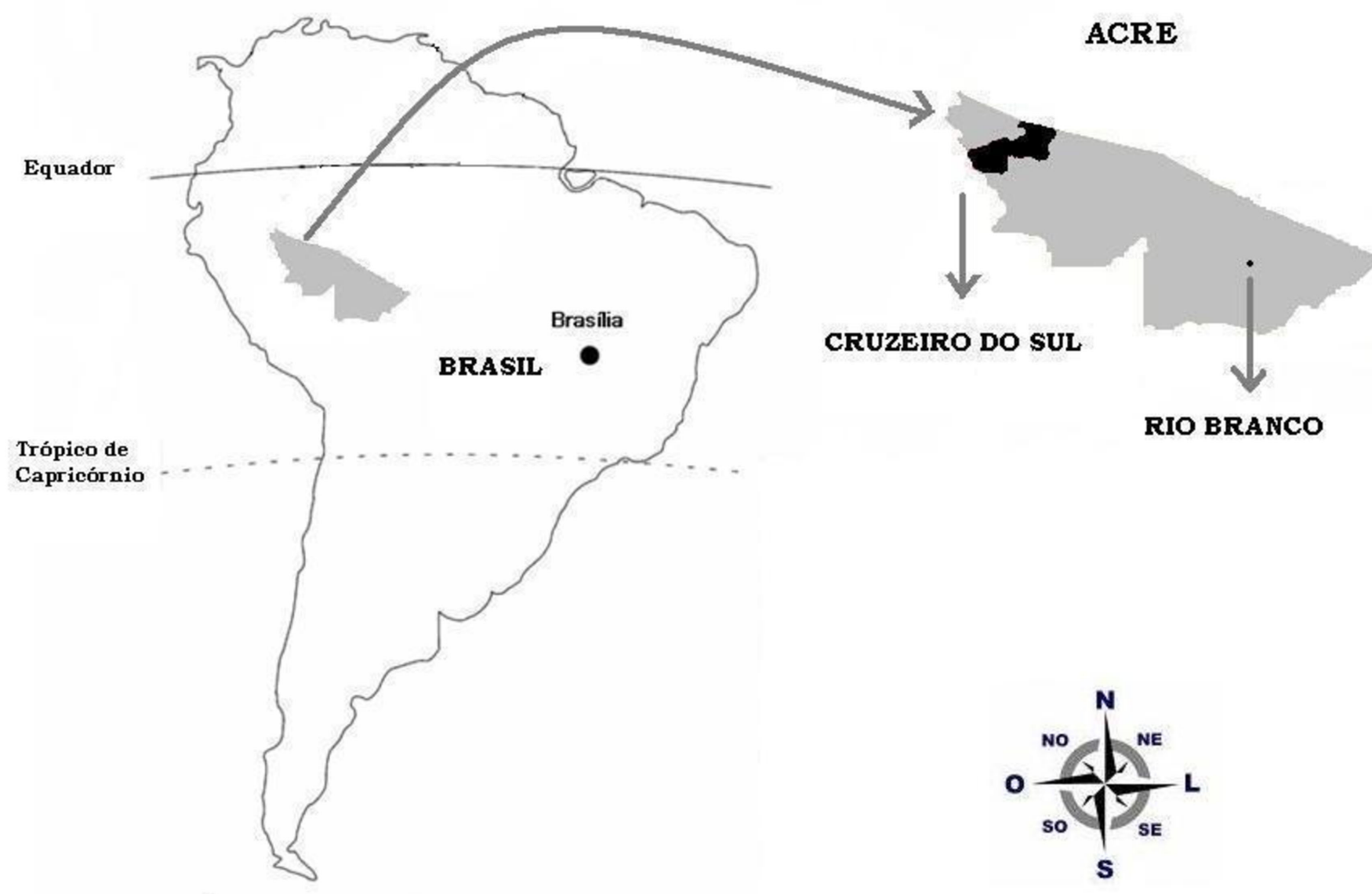

AMÉRICA DO SUL 


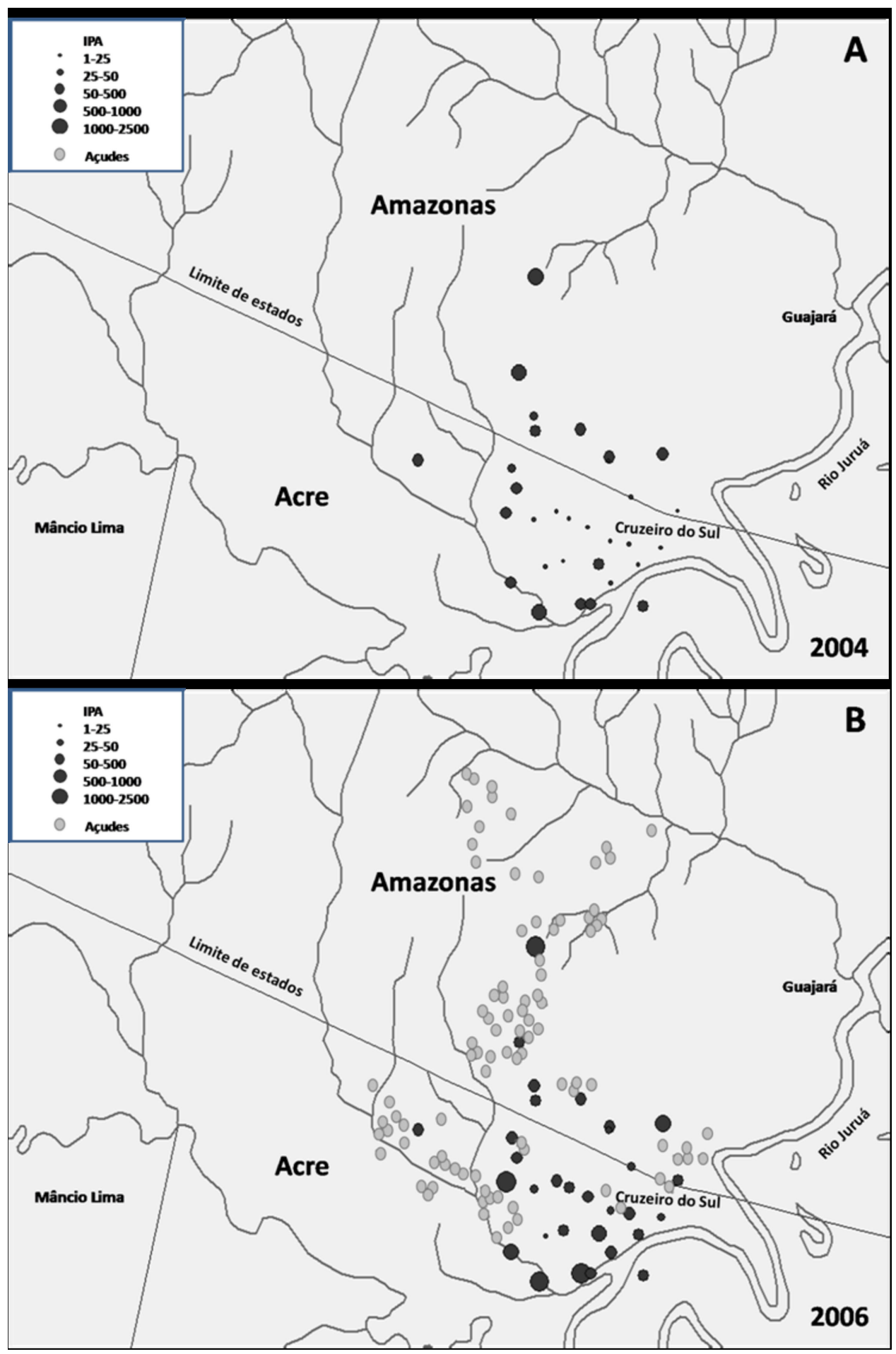

